Pensamiento Crítico Vol. 19 № 1, pp. 115-122

\title{
Economía política para entender el crecimiento económico en China
}

Political economy to understand the economic growth in China

Carlos Palomino Selem*

\section{RESUMEN}

Después de un excelente perfil de crecimiento económico en China durante la primera década del siglo XXI, este país experimenta una crisis de modelo de acumulación. El presente documento tiene por objeto desarrollar un marco teórico que parte de un esquema que emplea los criterios de la economía política para explicar que el problema por el cual atraviesa el gigante del Asia, no se resolverá recurriendo principalmente a las políticas convencionales de tipo macroeconómico monetario, concernientes a las reformas estructurales requeridas.

Palabras clave: cosmovisión, pensamiento económico, teoría económica, enfoque positivista, tomadores de decisión económica, ideológica, pensamiento no ortodoxo, institucional, economía mixta, capital estatal, riesgo crediticio.

\begin{abstract}
After a profile of excellence economic growth in China during the first decade of XXI century; this country is experiencing a crisis of accumulation model. This paper aims to develop a theoretical framework of a scheme employing the criteria of political economy to explain the problem which crosses the giant Asian
\end{abstract}

* Prof. dpto. Economía UNMSM. Director de Grossman Capital Markets financial advisor para Interactive Brokers - U.S. 


\section{Pensamiento Crítico Vol. 19. N I}

country, the pampering that will not be solved using mainly conventional policies macroeconomic and / or monetary and so-called structural reforms required.

Keywwords: Weltanschauung, Economic Thinking, Economic Theory, Positivism, Economic maker decisions, Ideological, Non ortodoxia thinking, Institutional, Mixed economy, Financial capital, Default Risk

\section{Economía política y weltanschauung para China}

Uno de los grandes problemas que sigue enfrentando el pensamiento económico actual se encuentra en el enfoque positivista o neopositivista lógico, el cual es asumido por una corriente de pensamiento importante dentro de la teoría económica; así es que llegamos a ser partícipes del debate planteado entre esta conceptualización de la realidad económica y el pensamiento que surgió con el nacimiento de la teoría económica conocida como economía política.

En términos gnoseológicos el planteamiento del problema es fundamental. En efecto, desde el punto de vista de la teoría del conocimiento, el positivismo como metodología es reduccionista, estudia solo el evento económico desvistiéndolo de su contenido político-social que lo sostiene. Esto entraña abordar una realidad compleja, como la organización económico-social, de manera simplista; la cual se construye dentro de una realidad más completa que es la realidad sociocultural, como la denominó el economista inglés K. Boulding (1956).

Ubicado dentro del contexto positivista, los tomadores de decisiones económicas (maker decisions) son entes neutrales cuya racionalidad obedece solo a criterios científico-técnicos sustentados en principios objetivos, abstractos, totalmente carentes de mayor sentido y dirección (problema de las teleologías). Pero, como sabemos, los tomadores de decisiones no solo se encuentran sujetos a restricciones de tipo social, cultural y política; sino que son entes a los que corresponden concepciones ideológicas, las cuales se vinculan mejor o más próximas a un sector social-productivo y a un modelo económico que hace viable a las clases que lo refrendan. En este sentido, consciente o inconscientemente se puede estar cayendo en este sesgo. 


\section{Carlos Palomino Selem}

El surgimiento del pensamiento no ortodoxo en economía ha tomado vigor en los ultimos años, debido a las limitaciones del positivismo lógico.

El problema del positivismo como método de conocimiento sobre el ser radica en que segmenta la realidad reduciendo su objeto de estudio a un aspecto de ella. En el caso de la economía lo abarca solo en el hecho económico; por ejemplo, analizar la formación de los precios como si se tratara de un evento que solo implicara un hecho basado en una racionalidad individual que se encuentra desvestida del contexto en la cual se encuentra el aquí-y-ahora, así como el histórico del cual proviene.

En este sentido, se aborda el crecimiento económico como si se tratara de una política dada por un ente sin rostro, como decir: "El país tal cayó en un modelo ya superado de intervención en la economía, que condujo a problemas de bajas tasas de crecimiento, baja productividad de la mano de obra, inflación y desempleo...", esto hace parecer que la política aplicada en dicho país fuera diseñada y llevada a cabo por "generación espontánea" producto de malos técnicos o políticos; es decir, se ha colocado un velo sobre quién, por qué y qué motivación llevó a diseñar y aplicar aquella política en el mencionado país. Desconociendo esto, no podemos resolver el problema de manera integral.

Bajo el debate entre economía positivista y economía política, planteamos el problema de China, como un problema de tipo político; consideramos que China no ha dejado de ser una economía mixta y que desea profundizar este modelo. En efecto, despues de renunciar al socialismo en el primer quinquenio de los 70 del siglo XX, una parte importante de la economía se encuentra gobernada por empresas estatales ubicadas en sectores estratégicos, con formación de capital financiero para su crecimiento que proviene del Estado. A a este tipo de recursos financieros se le denomina "capital estatal".

En China, el Estado controla en general las grandes empresas (Ceballos 2008) y, por tanto, las empresas que se desempeñan en los sectores considerados estratégicos (Enríquez 2012); es decir, el Partido Comunista de China (PCC) es el principal tomador

de decisiones económicas, productivas y comerciales. En este contexto, China carece de una clase capitalista independiente con capacidad de liderar la toma de decisiones. 


\section{Pensamiento Crítico Vol. 19. N I}

Incorporando el marco monetario a esta sección de nuestro análisis, el Banco del Pueblo de China, el banco central de dicho país, forma parte del esquema de desarrollo estratégico de este. Los mercados financieros, que incluyen el monetario de tipos de interés, cambiario y financiero de créditos, se han estructurado para conseguir una correspondencia necesaria que sustente el modelo estratégico de crecimiento que hasta hoy acompañó a China.

\section{Una aproximación al modelo de crecimiento económico chino}

El crecimiento económico de China se sustenta en el consumo externo de bienes industriales manufacturados de las economías capitalistas desarrolladas, como Estados Unidos y Europa Occidental (desarrollada), mercados de tamaño grande. Muy a pesar de los años de crecimiento con tasas por encima del 9\%, desde el 2000 hasta el 2007 (Quiroga 2009), el modelo no consiguió forjar un mercado interno sobre el cual pueda consolidar un crecimiento autónomo no dependiente del exterior.

Los principales compradores a nivel mundial de China, entre el 2000 y 2007 (pre crisis financiera del 2008) fueron los Estados Unidos y la Unión Europea (UE); de tal manera que cuando dicha crisis se expresa plenamente en septiembre del 2008, con la caída de Lehman Brothers en noviembre se aprecia que por primera vez, luego de 7 años, la reducción en el crecimiento de las exportaciones chinas, lo cual trajo con esto la quiebra de compañías en la región costera del país (Quiroga 2009).

Este consumo excesivo de los Estados Unidos y la UE fue financiado de forma importante por las economías emergentes, particularmente por China; así el país asiático se constituyó en el primer comprador de instrumentos del Tesoro de los Estados Unidos (ver gráfico 1 ). 


\section{Carlos Palomino Selem}

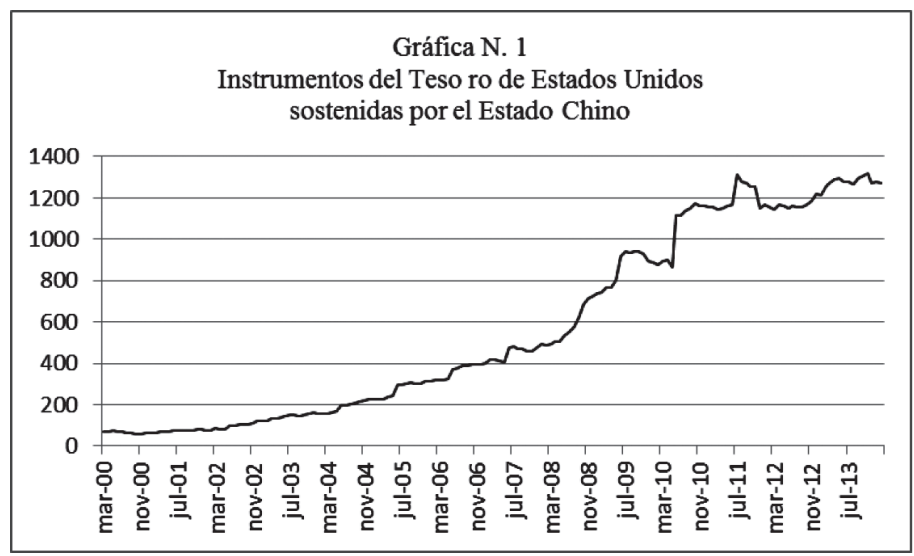

Fuente: Departamento del Tesoro de los Estados Unidos de América. Elaboración propia.

Pero algo más significativo resulta en el hecho de que en el proceso de acumulación internacional, las economías capitalistas desarrolladas han asignado a China un rol dentro de la división del trabajo a escala mundial. En efecto, el país del Asia produce y exporta los bienes industriales manufacturados que producían aquellas a mediados del siglo pasado (Ceballos 2008); y para ello cuenta con un importante recurso que es la mano de obra.

Así China se constituyó en fuente de recursos para las economías desarrolladas capitalistas. Por el lado, de la mano de obra, China es una oferta de mano de obra barata (Rojas 2013); y por otro lado, fue una fuente de financiamiento para dichos países. Los bajos costos laborales en China animó a que las corporaciones empresariales no financieras de Estados Unidos y la UE desplazaran su producción hacia el país asiático.

Mirando la remuneración anual, no el ingreso percápita para evitar distorsiones de tamaño poblacional; en el gráfico 2 se aprecia el salario anual en distintos países, donde el costo de mano de obra resulta menor, incluso en relación con las economías emergentes de menor tamaño comercial que China. 


\section{Pensamiento Crítico Vol. 19. N I}

Gráfico 2 Salarios anuales año 2005

\begin{tabular}{|l|l|l|c|}
\hline $\begin{array}{l}\text { Países no } \\
\text { Emergentes }\end{array}$ & Salarios & Países Emergentes & Salarios \\
\hline Reino Unido & 36,507 & Polonia & 5,513 \\
\hline Alemania & 36,242 & Malasia (2001) & 4,835 \\
\hline Japón (2005) & 32,909 & Marruecos & 3,386 \\
\hline Canadá & 28,241 & Brasil (2002) & 3,010 \\
\hline $\begin{array}{l}\text { Estados } \\
\text { Unidos (2003) }\end{array}$ & 27,507 & Filipinas (2003) & 2,412 \\
\hline España & 17,547 & China & 1,937 \\
\hline
\end{tabular}

Fuente: "El ascenso económico de China: Implicancias estratégicas para la seguridad global", P. Bustelos - OIT.

En el campo cambiario, el modelo chino ha tenido su expresión en la defensa del yuan (su moneda). Así, con el propósito de mantener las exportaciones a nivel alto, se requería que el dólar se apreciara respecto al yuan; la lógica se encuentra en que las mayores exportaciones hacían que se presente una abundancia relativa de dólares americanos (USD), lo cual haría que el USD se devaluara. Pero para que esto no afecte los costos de producción de China en USD, haciendo al país menos competitivo respecto al exterior, el Banco Central de China compraba los dólares para que las exportaciones no cayeran.

El mercado de dinero (créditos) y capitales en China también requiere de profundas modificaciones estructurales. En el mercado de créditos, ha surgido una banca en la sombra, la cual es uno de los principales mecanismos de financiación para la mediana empresa. Esta banca opera fuera de los registros de la banca formal, es una banca informal (no regulada como un banco) que procesa créditos con alto riesgo crediticio (default risk), que ha encontrando alivio a sus problemas de liquidez en el banco central. De este hecho es que los reportes (operaciones monetarias) se hayan convertido en un importante mercado que marca los precios del dinero.

En este segmento del mercado monetario-crediticio (incluye a la banca en la sombra), la tasa de interés ha experimentado alta volatilidad, llegando hasta el $30 \%$ (BIS 2013). Dicha tasa se convierte hoy en un factor de riesgo para la recuperación macroeconómica. 


\section{Carlos Palomino $\mathbf{S e l \in m}$}

De otra parte, en la sesión III de la reunión del Buró Político del PCC en diciembre del 2013, se enfatizó la necesidad de realizar una reforma integral en el campo económico, la cual se refería a la necesidad de abrir importantes empresas estatales al capital financiero privado, haciendo para ello que extranjeros compren acciones de dichas empresas estatales, configurando así un "sistema de propiedad mixta" dentro de una economía socialista al "estilo de China".

El Estado chino no dispone del capital financiero exigido para continuar el crecimiento económico que tiene esperado. Esto requiere de una reforma estructural en el mercado de capitales, lo cual se encuentra pendiente en términos de incentivos y negociación para hacerlo viable: factible y deseado. Pero, más aún, está el problema que este capital financiero no encuentre contradicciones con sus intereses en la estrategia global delPCC y su relación con el capital privado.

\section{Conclusiones}

Desde una perspectiva de la economía política, a China le será ineludible resolver la pregunta respecto a quién dirigirá la economía de dicho país en el futuro, de manera que se cumpla con los objetivos de crecimiento económico, pero esta vez replanteando su rol dentro del escenario mundial. La búsqueda de una mayor participación de inversionistas extranjeros llamados a ingresar flujo de fondos hacia las empresas del país (influjo) a través de las compras de acciones (participaciones) en compañías chinas pondrá en dilema el rol y las relaciones entre esta clase de capitalistas y el PCC en las principales decisiones de tipo económico. Hoy el PCC posee la supremacía y lidera la toma de decisiones en términos político-económicos.

Esta apertura de la economía china hacia una economía con mayor participación de capital privado, y no necesariamente chino, nos conduce a analizar los términos en que China replantearía su participación dentro del esquema de desarrollo político y económico a nivel mundial, dentro del esquema de división internacional del trabajo y organización de bloques que se tiene hoy.

En esta dirección, consideramos que si bien bajo un enfoque económico se tratara solo de ciertos ajustes de tipo macroeconómico-monetario, entonces la reactivación 


\section{Pensamiento Crítico Vol. 19. N I}

de la economía china es cuestión de tiempo, para observar mejoras en los mercados de metales y otros. Pero esta es una visión limitada del acontecer; la reestructuración económica tomaría un tiempo mayor con la incertidumbre que trae esta.

\section{Referencias bibliográficas}

BANCO INTERNACIONAL DE PAGOS; 2013; "Volatilidad interbancaria en China"; en Informe Trimestral del del Banco Internacional de Pagos (Bank for International Settlements): Los mercados precipitan un endurecimiento

BUILDING, Kenneth; 1956; en "System Thinking System Practice" de Peter Checklnad; 1ra. Edición; Editorial John Wiley \& Sons.

BUSTELOS, Pablo; 2009; "El ascenso económico de China: Implicancias estratégicas para la seguridad global; documento de la Oficina Internacional del Trabajo (OIT)

CEBALLOS, Miguel; 2008; La República Popular China: La última revolución industrial; China: una necesidad para una empresa global; Cátedra Nebrija-Grupo Santander.

DEPARTAMENTO DEL TESORO US; 2014; http://www.treasury.gov/ticdata/Publish/ mfhhis01.txt.

ENRÍQUEZ, Isaac; 2012; Notas para una interpretación de las estrategias de desarrollo en China: las funciones del Estado en la economía y en la consecución del "milagro económico" chino; Revista Economía crítica y crítica de la economía; Número 4, abril-mayo.

QUIROGA, Gloria; 2009; "China 30 de crecimiento económico"; Anuario Jurídico y Económico Escurialense XLII.

ROJAS, Carlos; 2013; La estrategia de BPO \& tercerización, ¿una opción de servicios para Colombia?; file://C:/Users/Carlos/Downloads/1770-1998-1-PB.pdf 